\title{
Self-shape optimisation principles: Optimisation of section capacity for thin-walled profiles
}

\author{
Benoit. P. Gilbert ${ }^{(1)}$, Lip H. Teh ${ }^{(2)}$, Hong Guan ${ }^{(3)}$ \\ (1) Lecturer, Griffith School of Engineering, Gold Coast Campus, Griffith University, QLD 4222, Australia, \\ Email: b.gilbert@griffith.edu.au, Phone: +61 755528577 (corresponding author) \\ (2) Senior Lecturer, School of Civil, Mining and Environmental Engineering, University of Wollongong, NSW \\ 2522, Australia, Email: Iteh@uow.edu.au \\ (3) Associate Professor, Griffith School of Engineering, Gold Coast Campus, Griffith University, QLD 4222, \\ Australia, Email: h.guan@griffith.edu.au
}

\section{ABSTRACT}

For economical benefits, optimisation of mass-produced structural steel products has been widely researched. The objective is to minimise the quantity of material used without sacrificing the strength and practicality of the structural members. Current research focuses on optimising the main dimensions of conventional cross-sectional shapes but rarely considers discovering new optimum shapes. This paper introduces the concepts of a new optimisation method that enables the crosssection to self-shape to an optimum by using the evolution and adaptation benefits of Genetic Algorithm (GA). The feasibility and accuracy of the method are verified by implementing it to optimise the section capacity of thin-walled profiles. Specifically, the profiles are optimised against simple parameters for which analytical solutions are known, namely the optimisation of doublysymmetric closed profiles. Results show that the cross-section accurately self-shapes to its optimum in a low number of generations. Factors influencing the convergence are presented in this paper. The method is extended to optimisation of cold-formed steel open section columns in the companion paper.

\section{KEYWORDS}

Optimisation, Genetic Algorithm, Thin-walled structures, Cold-formed steel structures 


\section{INTRODUCTION}

\subsection{General}

Cold-formed steel profiles are manufactured by bending a thin sheet of steel to a desired shape allowing efficient profiles to be used where conventional hot-rolled steel profiles prove uneconomic [1]. They are mass-produced and commonly used in applications such as steel storage racks, roof and wall systems, composite concrete and steel slabs, or automotive parts.

One of the main advantages of cold-formed steel profiles is the great flexibility of their crosssectional shapes, attributable to the manufacturing process allowing achievement of almost any desired cross-section. The cross-sectional shape is the key element in enhancing the strength of cold-formed steel profiles as it controls the three fundamental buckling modes: local, distortional (for open profiles) and global. However, research on optimisation of cold-formed steel profiles has been restricted mainly to the conventional $C, Z$ or $\Sigma$ cross-sectional shapes [2-9] as shown Figure 1. Web and/or flange stiffeners (see Figure 1) used to avoid local instabilities were sometimes considered in the optimisation process. In these attempts the search areas were restricted, as new cross-sectional shapes were not considered, and only the dimension variables of the existing cross-sections were optimised (height, width and thickness). Therefore innovations are very limited.

One can genuinely ask if cross-sectional shapes better than the conventional ones exist. Could one allow the cross-section to shape automatically in a natural way to an optimum without being restricted to a narrow search area?

This paper introduces the concepts of a new optimisation method that enables the cross-section to self-shape to an optimum by using the evolution and adaptation benefits of Genetic Algorithm. The feasibility and accuracy of the method are verified by implementing it to optimise the section capacity of thin-walled profiles. Specifically, the profiles are optimised against simple parameters for which analytical solutions are known. This paper constitutes the initial phase of a project aiming at finding practical and innovative cold-formed steel profiles without presumptions for the cross- 
sectional shapes, an optimisation process referred to as "shape discovery" [10]. Factors influencing the convergence are also presented herein.

The method is extended in the companion paper [11] to the optimisation of cold-formed steel columns by introducing the three fundamental modes of buckling for cold-formed steel members in the algorithm: local, distortional and global.

\subsection{Literature review, GA and "shape discovery"}

Initially developed by John Holland [12] in the 1960s, Genetic Algorithm (GA) mimics the Darwin evolution theory of "survival of the fittest". GA enables searching optimum solutions efficiently, is suitable for highly non-linear problems, and does not require solving complex optimisation equations. GA is intended for unconstrained optimisation problems, although constraints (involved in most real world problems) are commonly introduced into the "fitness function" as penalty functions, as outlined in Section 2. GA has been successfully applied to a vast range of engineering and science disciplines $[13,14]$, the literature featuring a wealth of articles demonstrating that GA can be an efficient and powerful optimisation method. In structural engineering, GA has been used to optimise frames and trusses [15-17] or steel and concrete structures [18, 19], although only a limited number of parameters were optimised in these attempts.

Lu and Makelainen [20-22] and Lee et al. [6, 7] used GA to optimise cold-formed steel hat, C and $\Sigma$ profiles. However, in these works, GA was used as a traditional optimisation method, and only the dimensions of the profiles were optimised.

Research involving optimisation of un-predefined cross-sections have been carried out successfully by Griffiths and Miles [10] for hot-rolled steel profiles and Liu et. al. [23] for cold-formed steel profile. Griffiths and Miles [10] used GA and a voxel-based representation in which the design space was decomposed into a grid of identical sized squares. GA cross-over and mutation operators were not applied to the genotype strings but to the design space, allowing evolution and convergence to known optimum I and box profiles. Liu et al. [23] used a "knowledge-based global optimisation" which found promising cross-sections through the knowledge-based optimisation 
process, and further optimised using a gradient-based local optimisation process. The sections were limited to eight folds, and minor stiffeners adding strength to the profiles were not considered.

Recently, Leng et al. [24] optimised the cross-sectional shapes of cold-formed steel open columns using three different optimisation algorithms, including the traditional GA. Sections having a wall thickness of $1 \mathrm{~mm}$ and a perimeter of $280 \mathrm{~mm}$ were divided into 21 elements (i.e. an element width of $13.33 \mathrm{~mm}$ ), and optimum "open circular" and "S" cross-sections were found. As in the case of Liu et al. [23], the length of the elements (about 14 times the profile thickness) may not allow small bending radii in the cross-sections and minor stiffeners to be created.

\section{OPTIMISATION PROBLEM}

Ragnedda and Serra [25] analytically investigated the optimum cross-section of a doubly symmetric thin-walled closed profile by minimising the cross-sectional area $A_{s}$ for imposed second moments of area $I_{x}$ and $I_{y}$ about the two axes of symmetry. They showed that the optimum crosssection is an ellipse and therefore a circle of radius $r$ if the two second moments of area $I_{x}$ and $I_{y}$ are equal. The radius $r$ is given as,

$$
r=\sqrt[3]{I_{x} / \pi t}
$$

where $t$ is the wall thickness.

The feasibility and accuracy of the self-shape optimisation method proposed in this paper are verified herein by implementing it on the previous well known optimisation problem. As this particular problem is doubly-symmetric, only a quarter of the cross-section needs to be considered. The constrained optimisation problem, consisting of minimising the cross-sectional area $A_{s}$ of the profile for given $I_{x}$ and $I_{y}$, can be transformed into an unconstrained problem suitable for GA as,

$$
\text { Minimise } f=\frac{A_{s}}{A_{\text {optimum }}}+\alpha_{x}\left|\frac{I_{s x}}{I_{x}}-1\right|+\alpha_{y}\left|\frac{I_{s y}}{I_{y}}-1\right|
$$


where $A_{\text {optimum }}$ is the known optimum cross-sectional area, $I_{s x}$ and $I_{s y}$ are the second moments of area of the profile about the two axes of symmetry, $\alpha_{x}$ and $\alpha_{y}$ are the penalty factors. $f$ is referred to as the fitness function and includes both the objective and the penalty functions. In the present case, the objective function, $A_{s} / A_{\text {optimum }}$, is chosen so $f$ converges to 1.0 for any values of the imposed second moments of area $I_{x}$ and $I_{y}$.

\section{SELF-SHAPE OPTIMISATION PRINCIPLE}

The main characteristics of the self-shape optimisation principles are:

- The initial population in GA is generated by arbitrarily drawing cross-sections using selfavoiding random walks in a design space of dimension $x_{\max } \mathrm{mm}$ by $y_{\max } \mathrm{mm}$, as detailed in Section 3.2. The self-avoiding random walks enable the generation of cross-sections without presumptions of their shapes.

- Elitism is included in the optimisation process, and the best two cross-sections of one generation are automatically copied to the next generation [22].

- A floating-point type GA [26] is used in this research, meaning that a cross-section is not represented by typical binary strings but by floating-point numbers representing the coordinates of the points constituting the cross-section as,

$$
\left[\begin{array}{l}
X_{i} \\
Y_{i}
\end{array}\right]=\left[\begin{array}{l}
0, x_{i, 2}, \ldots, x_{i, n i-1}, x_{i, n i} \\
y_{i, 1}, y_{i, 2}, \ldots, y_{i, n i-1}, 0
\end{array}\right]
$$

where $\left[X_{i}, Y_{i}\right]$ is the coordinate vector of the $i^{\text {th }}$ cross-section with $x_{i, j}$ and $y_{i, j}$ being the $x$ and $y$ coordinates, respectively, of the $f^{\text {th }}$ point of the cross-section. As only a quarter of the doublysymmetric cross-section is analysed, the first point and the last point of the cross-section are forced to lie on the $y$ and $x$-axes respectively.

- Cross-over and mutation operators are performed in relation to the design space and not to the floating-point variables, as detailed in Sections 3.3 and 3.4. 
- Half of the population is allowed to enter the mating pool and the roulette wheel selection method is used.

A flowchart of the global algorithm is presented in Figure 2 with specific operations detailed in Sections 3.1 to 3.4 .

\subsection{Augmented Lagrangian method}

Augmented Lagrangian method for GA has proven to be a powerful tool $[20,27]$ to avoid an illconditioned process by ensuring finite values of penalty factors. The method described in Adeli and Cheng [27] is used herein and the optimisation problem given in Section 2 is expressed as,

$$
\text { Minimise } g=\frac{A_{s}}{A_{\text {optimum }}}+\frac{1}{2}\left(\gamma_{x}\left(\left|\frac{I_{s x}}{I_{x}}-1\right|+\mu_{x}\right)^{2}+\gamma_{y}\left(\left|\frac{I_{s y}}{I_{y}}-1\right|+\mu_{y}\right)^{2}\right)
$$

where $\gamma_{x}$ and $\gamma_{y}$ are the penalty function coefficients and $\mu_{x}$ and $\mu_{y}$ are real parameters associated with each equality constraint. Unless mentioned otherwise, initial values of $\gamma_{x}=\gamma_{y}=2$ are used in this paper (see Section 4.1.3 for the influence of the penalty function coefficients on the convergence of the algorithm). Initial values of $\mu_{x}=\mu_{y}=0$ are used as recommended in [28].

Unless mentioned otherwise, a penalty increasing constant $\beta=1.05$ is used to avoid premature convergence due to high values of $\beta$ (see Section 4.1 .2 for the influence of penalty increasing constant on the convergence of the algorithm). The convergence rate $\alpha$ is set to 1.5 [27].

The augmented Lagrangian method consists of the following steps [27]:

Step 1: Initialise the penalty function coefficients, $\gamma_{x}=\gamma_{y}=2$, the real parameters associated with each equality constraint, $\mu_{x}=\mu_{y}=0$ and the violation constraint Viol previous $=\infty$. Set the penalty increasing constant $\beta=1.05$, the convergence rate $\alpha=1.5$ and the terminating criteria $\varepsilon=10^{-6}$.

Step 2: Run the GA (see Figure 2) and obtain the second moments of area $I_{s x, \text { best }}$ and $I_{s y, \text { best }}$ of the fittest cross-section determined from Eq. (4). 
Step 3: Calculate the current violation constraint Viol $I_{\text {current }}=\max \left(\left|\frac{I_{s x, \text { best }}}{I_{x}}-1,,\right| \frac{I_{s y, \text { best }}}{I_{y}}-1 \mid\right)$

Step 4: Check convergence of the algorithm as,

a) If $\mathrm{Viol}_{\text {current }}<\varepsilon$, terminate the search process, the fittest solution found in Step 2 is the solution;

b) Else if Viol $_{\text {current }}>$ Viol $_{\text {previous, }}$ go to Step 5;

c) Else if Viol $_{\text {current }} \leq$ Viol $I_{\text {previous, }}$ go to Step 6 .

Step 5: Update the penalty function coefficients and real parameters associated with each equality constraint as,
a) If $\left|\frac{I_{s x, \text { best }}}{I_{x}}-1\right| \geq \frac{\text { Viol }_{\text {previous }}}{\alpha}$ do $\gamma_{x}=\beta \gamma_{y}$ and $\mu_{x}=\frac{\mu_{x}}{\beta}$
b) If $\left|\frac{I_{s y, \text { best }}}{I_{y}}-1\right| \geq \frac{\text { Viol }_{\text {previous }}}{\alpha}$ do $\gamma_{y}=\beta \gamma_{y}$ and $\mu_{y}=\frac{\mu_{y}}{\beta}$

c) Go to Step 2.

Step 6: Update the penalty function coefficients and real parameters associated with each equality constraint as,

a) $\mu_{x}=\mu_{x}+\left|\frac{I_{s x, \text { best }}}{I_{x}}-1\right|$ and $\mu_{y}=\mu_{y}+\left|\frac{I_{s y, \text { best }}}{I_{y}}-1\right|$

b) If Viol $_{\text {current }} \geq \mathrm{Viol}_{\text {previous }} / \alpha$ then,

b1) If $\left|\frac{I_{s x, \text { best }}}{I_{x}}-1\right| \geq \frac{\text { Viol }_{\text {previous }}}{\alpha}$ do $\gamma_{x}=\beta \gamma_{x}$ and $\mu_{x}=\frac{\mu_{x}}{\beta}$
b2) If $\left|\frac{I_{s y, \text { best }}}{I_{y}}-1\right| \geq \frac{\text { Viol }_{\text {previous }}}{\alpha}$ do $\gamma_{y}=\beta \gamma_{y}$ and $\mu_{y}=\frac{\mu_{y}}{\beta}$

c) $V i o I_{\text {previous }}=V_{\text {Viol }} I_{\text {current }}$ and go to Step 2.

Steps 3 to 5 are referred to as the inner loop and Step 6 is referred to as the outer loop.

As developed above, the penalty function coefficients, $\gamma_{x}$ and $\gamma_{y}$, and the real parameters associated with each equality constraint, $\mu_{x}$ and $\mu_{y}$, are regularly increased through the augmented Lagrangian method to ensure that the constraints are satisfied. This change of the values of $\gamma_{x}, \gamma_{y}, \mu_{x}$ 
and $\mu_{y}$ does not facilitate visualising the convergence of the algorithm if the fitness function $g$ in Eq. (4) is plotted. As a result and for clarity, the simpler fitness function $f$ in Eq. (2), with constant penalty factors $\alpha_{x}$ and $\alpha_{y}$, is adopted through this paper to visualise the convergence of the algorithm.

\subsection{Initial population}

Cross-sections are drawn using self-avoiding random walks on a $x_{\max } \mathrm{mm} \times y_{\max } \mathrm{mm}$ design space, based on the following rules allowing arbitrary and continuous cross-sections to be drawn:

Step 1: a) A random point is chosen on the vertical axis ( $y$-axis) in the interval $\left[0, y_{\max }\right]$ (see Figure $3(\mathrm{a}))$.

b) An element is created from the first built point in the direction randomly chosen between $-45^{\circ}$ to $45^{\circ}$ to the horizontal (see Figure $3(\mathrm{a})$ ).

Step 2: A new element is created from the last built element in the direction randomly chosen between $-45^{\circ}$ to $45^{\circ}$ to the orientation of the last built element (see Figure 3 (b)).

Step 3: Perform the following checks:

a) If the last built element intersects the cross-section (i.e. the cross-section is not selfavoiding) or the axes $x=0, x=x_{\max }$ or $y=y_{\max }$ (i.e. the boundaries of the design space), then delete that element and go to Step 2 (see Figure 3 (c)).

b) Else if the element intersects the axis $y=0$, then stop building the cross-section. The cross-section is considered to be valid and is added to the initial population.

c) Otherwise go to Step 2.

The nominal size of the elements is set to be $1 \mathrm{~mm}$, which corresponds to the wall thickness of all profiles used to validate the method in Section 4. The size of the elements and its relationship with the internal bending radius is discussed in Section 5 for future cold-formed steel applications.

Self-avoiding random walks can self-trap (see Figure 3 (d)), and a cross-section is considered self-trapped if Step 3(a) is repeated 10 times in a row. The cross-section is then considered unfit and not added to the initial population. Initial cross-section examples are shown in Figure 4 on a 40 $\mathrm{mm} \times 40 \mathrm{~mm}$ design space. 


\subsection{Cross-over operator}

A one-point cross-over operator type is used herein. Two points $P_{\text {parent } 1}$ and $P_{\text {parent2 }}$ are chosen at $\delta \%$ along the length of the first and second parents, respectively, with $\delta$ being a random number in the open interval ]0,100[, so the first and last points of the parents are not selected. Two points $P_{1}$ and $P_{2}$, as shown in Figure $5(\mathrm{a})$, are then defined using a linear interpolation between $P_{\text {parent } 1}$ and $P_{\text {parent2 }}$ as,

$$
P_{1}=\lambda P_{\text {Parent }}+(1-\lambda) P_{\text {parent }} \text { and } P_{2}=\lambda P_{\text {Parent }}+(1-\lambda) P_{\text {parent }}
$$

where $\lambda$ is a random number in the interval $[0,1]$. Eq. (5) allows new materials to be added in subsequent generations [26].

Two offsprings are created per operation with the first offspring built using the right-hand part of the first parent and the left-hand part of the second parent as,

Step 1: The right-hand part of the first parent is rotated about its intersection point $P_{y 1}$ with the yaxis and scaled so that the last point $P_{\text {parent } 1}$ of the part matches point $P_{1}$ as illustrated in Figure 5 (b).

Step 2: The left-hand part of the second parent is rotated about its intersection point $P_{x 2}$ with the xaxis and scaled so that the first point $P_{\text {parent2 }}$ of the part matches point $P_{1}$ as illustrated in Figure 5 (b).

Step 3: The two parts created in Steps 1 and 2 are added together in Figure 5 (b).

Step 4: Elements constituting the offspring are merged or subdivided to keep all elements about 1 $\mathrm{mm}$ long, in the interval [0.75 mm, $1.5 \mathrm{~mm}]$.

Step 5: If the created cross-section intersects itself, the offspring is considered unfit and is disregarded as illustrated in Figure 2.

The second offspring is similarly based on the left hand part of the first parent and the right hand part of the second parent using point $P_{2}$ instead of $P_{1}$ (see Figure 5 (c)). Offsprings are created until the overall population is replaced (see in Figure 2). A typical cross-over probability of 0.8 is used. 


\subsection{Mutation operator}

Mutation allows new cross-sectional shapes to be introduced into the population by redrawing a part or several parts of a cross-section. The operator acts on the points constituting the crosssections with a typical mutation probability of 0.01 for each point. If a point mutates, the part of the cross-section around that point is redrawn as,

Step 1: A number of elements is randomly chosen in the interval $[1,0.25 \times$ the number of elements constituting the cross-section] and deleted on each side of the mutated point (see Figure $6)$.

Step 2: A new arbitrary, self-avoiding and continuous shape is drawn in an infinite design space based on the principles detailed in Section 3.2 with the exception of Step 3(b) (see Figure 6). The number of elements constituting this shape is twice the number of elements chosen in Step 1.

Step 3: a) If the number of elements between the intersections of the initial cross-section with the x-axis (point $P_{x}$ ) or the $y$-axis (point $P_{y}$ ) is greater than the number of element chosen in Step 1, go to Step 4.

b) Else go to Step 5 .

Step 4: The created shape in Step 2 is inserted in the cross-section in lieu of the elements deleted in Step 1 (see Figure 6 (a)). Go to Step 6.

Step 5: a) A new point is defined on the $x$ - or $y$-axes at a distance $\pm d$ from point $P_{x}$ or $P_{y}$ respectively, where $d$ is randomly chosen in the interval $[0$, nominal size of one element $x$ (number of elements chosen in Step 1 - number of elements between the mutated point and point $P_{x}$ or $\left.\left.P_{y}\right)\right]$

b) The created shape in Step 2 is inserted in the cross-section between the new point defined in Step 5 a) and the first point of the cross-section not deleted in Step 1 (see Figure $6(b))$. 
Step 6: Elements constituting the mutated part are merged or subdivided to keep all elements about $1 \mathrm{~mm}$ long, in the interval [0.75 mm, $1.5 \mathrm{~mm}]$.

A number of elements deleted and redrawn equal to a maximum of $50 \%$ of the number of elements constituting the cross-section was found to appropriately allow new characteristics to be added to the cross-sections, as investigated in [29]. If the mutated cross-section intersects itself, the cross-section is considered unfit and is disregarded as shown in Figure 2. A new cross-section is then created from the cross-over and mutation operators to maintain the size of the population.

Step 5 allows cross-sections to intersect the $x$ - or $y$-axes at new locations and overcomes this deficiency in the cross-over operator. The further the mutated point is from the intersection points $P_{x}$ or $P_{y}$ of the cross-section with the $x$ - or $y$-axes, the closer the new point is to point $P_{x}$ or $P_{y}$.

\section{RESULTS}

\section{$4.120 \mathrm{~mm}$ radius circle}

A $20-\mathrm{mm}$ radius circle with $1 \mathrm{~mm}$ wall thickness is used in this section to validate the self-shape optimisation principle. The design space is set to be $40 \mathrm{~mm} \times 40 \mathrm{~mm}$, i.e. twice the radius of the circle.

When creating the cross-sections in Section 3.2, the longer the perimeter of the cross-section, the higher likelihood the cross-section is to self-trap and not be added to the initial population. Therefore, cross-sections with small areas are more likely to be represented than cross-sections with large areas in the initial population. To avoid this phenomenon, allow diversity in the initial population and improve the convergence rate [29], the initial cross-sections are deliberately generated to be evenly distributed in five categories, of 10 elements each, between 10 elements (cross-sectional area $=40 \mathrm{~mm}^{2}-$ about one third of the optimum cross-sectional area) and 60 
elements (cross-sectional area $=240 \mathrm{~mm}^{2}-$ about twice the optimum cross-sectional area). Values of $A_{\text {optimum, }} I_{x}$ and $I_{y}$ in Eq. (2) for the $20 \mathrm{~mm}$ radius circle are given in Table 1.

The influence of the population size, the penalty increasing constant $\beta$ and initial penalty function coefficients $\gamma_{x}$ and $\gamma_{y}$ in the Augmented Lagrangian method on the convergence of the algorithm is investigated in this Section.

\subsubsection{Influence of the population size}

Figure 7 plots the average fitness function $f$ given in Eq. (2) for 10 runs with penalty factors $\alpha_{x}=$ $\alpha_{y}=10$ for population sizes of 400,700 and 1000 cross-sections.

The average errors over 10 runs in cross-sectional area and second moments of area are given in Table 2. For all population sizes, the algorithm accurately finds the optimum circle with average errors over 10 runs at the $100^{\text {th }}$ generation ranging between $0.25 \%$ and $0.50 \%$ for the crosssectional area and between $0.01 \%$ and $0.04 \%$ for the targeted second moments of area, with the population size of 1000 cross-sections giving slightly better results. As seen in Figure 7, about 50 generations are needed for the algorithm to converge to a near optimum solution.

The size of the population has little influence on the accuracy and convergence of the algorithm and a population size of 700 cross-sections is therefore chosen for all further analyses.

Figure 8 shows the evolution of the cross-section for one solution and a population size of 700 cross-sections. For clarity the entire design space of $40 \mathrm{~mm} \times 40 \mathrm{~mm}$ is not represented in Figure 8 .

\subsubsection{Influence of the penalty increasing constant $\beta$}

The penalty increasing constant $\beta$ increases the weight of the equality constraints in Eq. (4) if the constraints are greater than a violation criterion, as shown in Section 3.1. The greater the constant $\beta$, the greater the increase in the weight of the equality constraints in the function $g$ in Eq. (4) [27]. This section investigates the accuracy of the algorithm for various values of $\beta$. 
Figure 9 plots the average fitness function $f$ given in Eq. (2) for 10 runs with penalty factors $\alpha_{x}=$ $\alpha_{y}=10$ and for penalty increasing constant $\beta$ ranging from 1.01 to 10 in the Augmented Lagrangian method.

The average errors over 10 runs in cross-sectional area and second moments of area are given in Table 3. For values of $\beta$ greater than 1.1, the algorithm tends to satisfy the equality constraints too rapidly and converges to local optima with an average error over 10 runs on the cross-sectional area of up to $8.94 \%$ for $\beta=10$. Typical solutions for $\beta=10$ at the $100^{\text {th }}$ generation are given in Figure 10.

The value of the penalty increasing constant $\beta$ is found to significantly influence the accuracy of the algorithm. A value of $\beta$ equal to 1.05 allows the algorithm to explore the design space before converging and is thus chosen for further analysis. This value of $\beta$ is significantly less than the value of 10 recommended by Belegundu and Arora [28] and Bazaraa et. al. [30].

\subsubsection{Influence of the penalty function coefficients $\gamma_{x}$ and $\gamma_{y}$}

Figure 11 plots the average fitness function $f$ given in Eq. (2) for 10 runs with penalty factors $\alpha_{x}=$ $\alpha_{y}=10$ and for penalty function coefficients $\gamma_{x}$ and $\gamma_{y}$ in the Augmented Lagrangian method ranging from 0.35 to 5 .

The average errors over 10 runs in cross-sectional area and second moments of area are given in Table 4. For all values of $\gamma_{x}$ and $\gamma_{y}$, the algorithm accurately finds the optimum circle with an average error over 10 runs at the $100^{\text {th }}$ generation of about $0.30 \%$ for the cross-sectional area and $0.03 \%$ for the targeted second moments of area. However, for small values of the penalty function coefficients $(0.35$ and 0.5$)$, the algorithm selects cross-sections having a relatively small number of elements as the fittest cross-sections in the first generations, because the initial weight of the penalty functions is much less that the weight of the objective function in Eq. (4). This selection continues until the real parameters $\gamma_{x}$ and $\gamma_{y}$ associated with each equality constraint are updated in the outer loop of the Augmented Lagrangian method presented Section 3.1. For values of $\gamma_{x}$ and $\gamma_{y}$ 
less than 0.35 , the algorithm does not converge and keep selecting cross-sections having a small number of elements as fittest cross-sections for all generations.

The value of the penalty function coefficients is found to influence the behaviour of the algorithm in the first generations and a value of $\gamma_{x}=\gamma_{y}=2$ is chosen for further analysis.

\subsection{Various radius circles}

The self-shape optimisation principle is checked in this section against optimum cross-sections corresponding to circles of radius ranging from $10 \mathrm{~mm}$ to $40 \mathrm{~mm}$. All cross-sections have a wall thickness of $1 \mathrm{~mm}$.

For each optimisation problem, the design space is set to be $2 r \times 2 r$, where $r$ is the radius of the circle. For the $10 \mathrm{~mm}$ radius circle, the cross-section areas in the initial population are uniformly distributed between zero and twice the cross-sectional area of the circle. For the remaining problems, they are uniformly distributed between $40 \mathrm{~mm}^{2}$ and twice the cross-sectional area of the corresponding circle. Values of $A_{\text {optimum }}, I_{x}$ and $I_{y}$ in Eq. (2) for all optimum circles are given in Table 1.

Figure 12 plots the average fitness function $f$ given in Eq. (2) for 10 runs with penalty factors $\alpha_{x}=$ $\alpha_{y}=10$ for the optimisation problems given above.

The average errors over 10 runs in cross-sectional area and second moments of area are given in Table 5. As the radius of the targeted optimum solution increases, the proposed GA keeps satisfying the constraints but moves away from the optimum cross-section. For the $40 \mathrm{~mm}$ radius circle, the average error for the cross-sectional area, over 10 runs, at the $100^{\text {th }}$ generation is still reasonable and equal to $1.63 \%$ with a coefficient of variation of 0.0032 . Figure 13 shows typical solutions for the $40 \mathrm{~mm}$ radius circle. For clarity, the entire design space in not shown in Figure 13.

The increase in the average error in the cross-sectional area with increasing radius $r$ of the targeted optimum solution is mainly related to the size of the design space and the number of elements constituting the cross-section. The size of the design space varies quadratically while the 
cross-sectional area varies almost linearly with the radius $r$. Therefore, as the value of $r$ increases, the algorithm has to investigate an increased number of solutions for a given accuracy.

The computational time is increased by a factor 11.3 between the $20 \mathrm{~mm}$ (32 elements) and the $40 \mathrm{~mm}$ (63 elements) radius circles with an average CPU time of 0.65 hour against 7.32 hours per run on a $3 \mathrm{GHz}$ Intel Core2 Quad processor. Detailed results are given in [29].

\subsection{Ellipses}

The self-shape optimisation principle is verified in the section against minimising the crosssectional area with different targets for the second moments of area about the two principal axes of bending. The optimum cross-section is an ellipse of radii (also referred to as semi-axes) $r_{x}$ and $r_{y}$ (see Section 2).

An ellipse of radii $r_{x}=30 \mathrm{~mm}$ and $r_{y}=15 \mathrm{~mm}$ with a wall thickness of $1 \mathrm{~mm}$ is used as the solution to the optimisation problem, with cross-sectional properties given in Table 6 . The design space is set to be $60 \mathrm{~mm} \times 30 \mathrm{~mm}$ and corresponds to twice the radii $r_{x}$ and $r_{y}$ of the ellipse, respectively. The initial population is uniformly distributed between cross-sectional areas of $40 \mathrm{~mm}^{2}$ (about one-third of the optimum cross-sectional area) and $240 \mathrm{~mm}^{2}$ (about twice the optimum crosssectional area).

Figure 14 plots the average fitness function $f$ given in Eq. (2) for 10 runs with penalty factors $\alpha_{x}=$ $\alpha_{y}=10$ for the optimisation problem given above.

The average errors over 10 runs in cross-sectional area and second moments of area are given in Table 7. The algorithm finds the optimum ellipse with average errors over 10 runs at the $100^{\text {th }}$ generation of $2.08 \%$ for the cross-sectional area and $0.05 \%$ for the second moments of area. The error in cross-sectional area is higher than those for the optimum circles, but still remains a reasonable level of accuracy. Figure 15 shows typical solutions at the $100^{\text {th }}$ generation. For clarity, the entire design space in not shown in Figure 15. 


\section{DISCUSION ON ELEMENT SIZE}

The computational time required to find the optimum $40 \mathrm{~mm}$ radius circle (63 elements) is equal to 7.32 hours per run on a $3 \mathrm{GHz}$ Intel Core2 Quad processor, 11.3 times that for finding the optimum $20 \mathrm{~mm}$ radius circle (32 elements). Therefore, a large number of elements constituting the cross-section may lead to unsatisfactory computational time and the number of elements must satisfy a compromise between being large enough to accurately draw the cross-section and small enough to allow reasonable computational cost.

Choosing an element size of twice the wall thickness would allow the internal bending radii to be as small as 0.91 times the wall thickness for a $90^{\circ}$ bend (see Figure $16(\mathrm{a})$ ). Such a radius is in the order of magnitude of the minimum bending radius specified in EN 10149-2 [31] for plates less than $3 \mathrm{~mm}$ thick. An element size of four times the wall thickness would allow the internal bending radii to be as small as 2.33 times the wall thickness for a $90^{\circ}$ bend (see Figure 16 (b)). This value is comparable to current industry practices and is about twice the minimum bending radius specified in the EN 10149-2 [31] for plates less than $3 \mathrm{~mm}$ thick.

An element size of 3.33 times the wall thickness is used in the companion paper [11] for the optimisation of cold-formed steel columns, which is found to be a reasonable compromise between accuracy and computational time.

\section{CONCLUSIONS}

This paper introduces the concept of a new optimisation procedure that allows arbitrary and continuously drawn cross-sections to self-shape to an optimum by using Genetic Algorithm and the Augmented Lagrangian method. The cross-over and mutation operators are described. The proposed method is checked against known solutions of minimising the cross-sectional area of thinwalled closed profiles for given second moments of area, and is found to accurately converge to 
these solutions. The method is extended in the companion paper [11] to the optimisation of coldformed steel columns. 


\section{REFERENCES}

[1] G.J. Hancock, Design of cold-formed steel structures (to AS/NZ 4600:2007) - 4th Edition, Australian Steel Institute, North Sydney, Australia, 2007.

[2] M. Ostwald, M. Rodak, Multicriteria optimisation of cold-formed thin-walled beams under compression axial load, in: K.J.R. Rasmussen, T. Wilkinson (Eds.) 5th International Conference on Coupled Instabilities in Metal Structures, Sydney, Australia, 2008, pp. 141-148.

[3] R.J. Kasperska, K. Magnucki, M. Ostwald, Bicriteria optimization of cold-formed thin-walled beams with monosymmetrical open cross sections under pure bending, Thin-Walled Structures, 45 (2007) 563-572.

[4] K. Magnucki, P. Paczos, Theoretical shape optimization of cold-formed thin-walled channel beams with drop flanges in pure bending, Journal of Constructional Steel Research, 65 (2009) $1731-1737$.

[5] K. Magnucki, M. Mackiewicz, J. Lewinski, Optimal design of a mono-symmetrical open cross section of a cold-formed beam with cosinusoidally corrugated flanges, Thin-Walled Structures, 44 (2006) 554-562.

[6] J. Lee, S.-M. Kim, H.-S. Park, B.-H. Woo, Optimum design of cold-formed steel channel beams using micro Genetic Algorithm, Engineering Structures, 27 (2005) 17-24.

[7] J. Lee, S.-M. Kim, H. Seon Park, Optimum design of cold-formed steel columns by using micro genetic algorithms, Thin-Walled Structures, 44 (2006) 952-960.

[8] A. Karim, H. Adeli, Global optimum design of cold-formed steel hat-shape beams, Thin-Walled Structures, 35 (1999) 275-288.

[9] M.M. Pastor, M. Casafont, E. Chillarón, A. Lusa, F. Roure, M.R. Somalo, Optimization of coldformed steel pallet racking cross-sections for flexural-torsional buckling with constraints on the geometry, Engineering Structures, 31 (2009) 2711-2722.

[10] D.R. Griffiths, J.C. Miles, Determining the optimal cross-section of beams, Advanced Engineering Informatics, 17 (2003) 59-76. 
[11] B.P. Gilbert, T.J.-M. Savoyat, L.H. Teh, Self-shape optimisation application: Optimisation of cold-formed steel columns, Thin-Walled Structures, (Submitted) (2012).

[12] J. Holland, Adaptation in Natural and Artificial Systems, University of Michigan Press, Ann Arbor, 1975.

[13] M. Gen, R. Cheng, Genetic algorithms and engineering design, New York, 1997.

[14] R. Haupt, S. Haupt, Practical genetic algorithms, New York, 1998.

[15] T. Xu, W. Zuo, T. Xu, G. Song, R. Li, An adaptive reanalysis method for genetic algortihm with application to fast truss optimization, Acta Mechanica Sinica, 26 (2010) 225-234.

[16] N. Bel Hadj Ali, M. Sellami, A.-F. Cutting-Decelle, J.-C. Mangin, Multi-stage production cost optimization of semi-rigid steel frames using genetic algorithms, Engineering Structures, 31 (2009) 2766-2778.

[17] M.N.S. Hadi, Y. Arfiadi, Optimum design of absorber for MDOF structures, ASCE Journal of Structural Engineering, 124 (1998) 1272-1280.

[18] A.M. El Ansary, A.A. El Damatty, A.O. Nassef, A coupled finite element genetic algorithm technique for optimum design of steel conical tanks, Thin-Walled Structures, 48 (2010) 260-273.

[19] Z. Aydin, Y. Ayvaz, Optimum topology and shape design of prestressed concrete bridge girders using a genetic algortihm, Structural and Multidisciplinary Optimization, 41 (2010) 151-162.

[20] W. Lu, P. Makelainen, Augmented Lagrangian genetic algorithms for optimal design of hatshaped cold-formed steel profile, in: 9th International Conference: Modern Building Materials, Structures and Techniques, Vilnius, Lithuania, 2007.

[21] W. Lu, P. Mäkeläinen, Fuzzy optimization of cold-formed steel sheeting using genetic algorithms, Journal of Constructional Steel Research, 62 (2006) 1276-1281.

[22] W. Lu, Optimum design of cold-formed steel purlins using genetic algorithms in: Laboratory of Steel Structures, Helsinki University of Technology, Helsinki, Finland, 2003.

[23] H. Liu, T. Igusa, B.W. Schafer, Knowledge-based global optimization of cold-formed steel columns, Thin-Walled Structures, 42 (2004) 785-801.

[24] J. Leng, J.K. Guest, B.W. Schafer, Shape optimization of cold-formed steel columns, ThinWalled Structures, 49 (2011) 1492-1503. 
[25] F. Ragnedda, M. Serra, On optimum thin-walled closed cross section, Structural and Multidisciplinary Optimization, 30 (2005) 233-235.

[26] H. Adeli, C.S. Kamal, Cost optimization of structures, Wiley, Chichester, England, 2006.

[27] H. Adeli, N.-T. Cheng, Augmented Lagrangian Genetic Algorithm for Structural Optimization, Journal of Aerospace Engineering, 7 (1994) 104-118.

[28] A.D. Belegundu, J.S. Arora, A computational study of transformation methods for optimal design, American Institute of Aeronautics and Astronautics Journal, 22 (1984) 535-542.

[29] F. Wald, Z. Sokol, J.-P. Jaspart, Base plate in bending and anchor bolts in tension, HERON, 53 (2008) 21-50.

[30] M.S. Bazaraa, H.D. Sherali, C.M. Shetty, Nonlinear programming, theory and algorithms, second edition, Wiley \& Sons, New-York, USA, 1993.

[31] EN 10149-2, Specification for hot-rolled flat products made of high yield strength steels for cold forming. Delivery conditions for thermomechanically rolled steels, European Committee for Standardization (CEN), Brussels, Belgium, 1996. 
Table 1: Characteristics of the optimum circles

\begin{tabular}{ccc}
\hline Radius $(\mathrm{mm})^{(1)}$ & $A_{\text {optimum }}\left(\mathrm{mm}^{2}\right)$ & $I_{X}, I_{Y}\left(\mathrm{~mm}^{4}\right)$ \\
\hline 10 & 62.8 & $3,149.5$ \\
20 & 125.7 & $25,148.4$ \\
30 & 188.5 & $84,846.6$ \\
40 & 251.3 & $201,093.4$ \\
\hline
\end{tabular}

(1): the radius is measured at the centreline of the wall thickness

Table 2: Average results at the $100^{\text {th }}$ generation for various population sizes

\begin{tabular}{c|cc|cc|cc} 
& \multicolumn{6}{|c}{ Results over 10 runs at the $100^{\text {th }}$ generation } \\
\hline $\begin{array}{c}\text { Population (number } \\
\text { of cross-sections) }\end{array}$ & $\begin{array}{c}A_{s} \\
\text { Aver. error (\%) }\end{array}$ & CoV & Aver. error $(\%)$ & CoV & Aver. error $(\%)$ & CoV \\
\hline 400 & 0.50 & 0.0023 & 0.02 & 0.0002 & 0.01 & 0.0001 \\
700 & 0.30 & 0.0015 & 0.02 & 0.0002 & 0.04 & 0.0005 \\
1000 & 0.25 & 0.0005 & 0.02 & 0.0002 & 0.02 & 0.0001 \\
\hline
\end{tabular}

Table 3: Average results at the $100^{\text {th }}$ generation for various $\beta$ factors

\begin{tabular}{c|cc|cc|cc} 
& \multicolumn{7}{c}{ Results over 10 runs at the $100^{\text {th }}$ generation } \\
\hline & \multicolumn{2}{|c|}{$A_{s}$} & \multicolumn{3}{c}{$I_{s x}$} & $I_{s y}$ \\
$\beta$ factor & Aver. error $(\%)$ & CoV & Aver. error (\%) & CoV & Aver. error $(\%)$ & CoV \\
\hline 1.01 & 0.31 & 0.0018 & 0.06 & 0.0010 & 0.07 & 0.0012 \\
1.05 & 0.30 & 0.0015 & 0.02 & 0.0002 & 0.04 & 0.0005 \\
1.1 & 0.51 & 0.0038 & 0.03 & 0.0002 & 0.01 & 0.0002 \\
1.5 & 2.98 & 0.0062 & 0.00 & 0.0000 & 0.00 & 0.0000 \\
2 & 4.43 & 0.0147 & 0.00 & 0.0000 & 0.00 & 0.0000 \\
10 & 8.94 & 0.0230 & 0.00 & 0.0000 & 0.00 & 0.0000 \\
\hline
\end{tabular}

Table 4: Average results at the $100^{\text {th }}$ generation for various penalty function coefficients

\begin{tabular}{c|cc|cc|cc} 
& \multicolumn{6}{c}{ Results over 10 runs at the $100^{\text {th }}$ generation } \\
\hline $\begin{array}{c}\text { Penalty function } \\
\text { coeff. } \gamma_{x} \text { and } \gamma_{y}\end{array}$ & $\begin{array}{c}A_{s} \\
\text { Aver. error }(\%)\end{array}$ & CoV & Aver. error $(\%)$ & CoV & Aver. error $(\%)$ & $I_{s y}$ \\
\hline 0.35 & 0.27 & 0.0010 & 0.01 & 0.0001 & 0.02 & 0.0002 \\
0.5 & 0.31 & 0.0017 & 0.02 & 0.0002 & 0.02 & 0.0001 \\
1 & 0.23 & 0.0013 & 0.01 & 0.0001 & 0.01 & 0.0002 \\
2 & 0.30 & 0.0015 & 0.02 & 0.0002 & 0.04 & 0.0005 \\
5 & 0.50 & 0.0015 & 0.03 & 0.0002 & 0.02 & 0.0002 \\
\hline
\end{tabular}

Table 5: Average results at the $100^{\text {th }}$ generation for various radius circles

\begin{tabular}{c|cc|cc|cc} 
& \multicolumn{6}{c}{ Results over 10 runs at the $100^{\text {th }}$ generation } \\
\hline $\begin{array}{c}\text { Optimum radius } \\
(\mathrm{mm})\end{array}$ & $\begin{array}{c}A_{s} \\
\text { Aver. error }(\%)\end{array}$ & CoV & Aver. error $(\%)$ & CoV & Aver. error $(\%)$ & CoV \\
\hline 10 & 0.24 & 0.0013 & 0.01 & 0.0001 & 0.01 & 0.0001 \\
20 & 0.30 & 0.0015 & 0.02 & 0.0002 & 0.04 & 0.0005 \\
30 & 0.87 & 0.0030 & 0.04 & 0.0006 & 0.04 & 0.0004 \\
40 & 1.63 & 0.0032 & 0.03 & 0.0002 & 0.07 & 0.0008 \\
\hline
\end{tabular}

Table 6: Characteristics of the optimum ellipse

\begin{tabular}{ccccc}
\hline$r_{x}(\mathrm{~mm})^{(1)}$ & $r_{y}(\mathrm{~mm})^{(1)}$ & $A_{\text {optimum }}\left(\mathrm{mm}^{2}\right)$ & $I_{x}\left(\mathrm{~mm}^{4}\right)$ & $I_{y}\left(\mathrm{~mm}^{4}\right)$ \\
\hline 30 & 15 & 141.4 & 18569.8 & 53035.0 \\
\hline
\end{tabular}

(1): the radius is measured at the centreline of the wall thickness

Table 7: Average results at the $100^{\text {th }}$ generation for a $30 \mathrm{~mm} \times 15 \mathrm{~mm}$ ellipse

\begin{tabular}{c|cc|cc|cc} 
& \multicolumn{6}{c}{ Results over 10 runs at the $100^{\text {th }}$ generation } \\
\hline $\begin{array}{c}\text { Ellipse } r_{x} \times r_{y} \\
(\mathrm{~mm} \times \mathrm{mm})\end{array}$ & \multicolumn{2}{|c|}{$A_{s}$} & \multicolumn{3}{c}{$I_{s x}$} & $I_{s y}$ \\
\hline $30 \times 15$ & Aver. error $(\%)$ & CoV & Aver. error (\%) & CoV & Aver. error $(\%)$ & CoV \\
\hline & 2.08 & 0.0017 & 0.04 & 0.0002 & 0.07 & 0.0006 \\
\hline
\end{tabular}




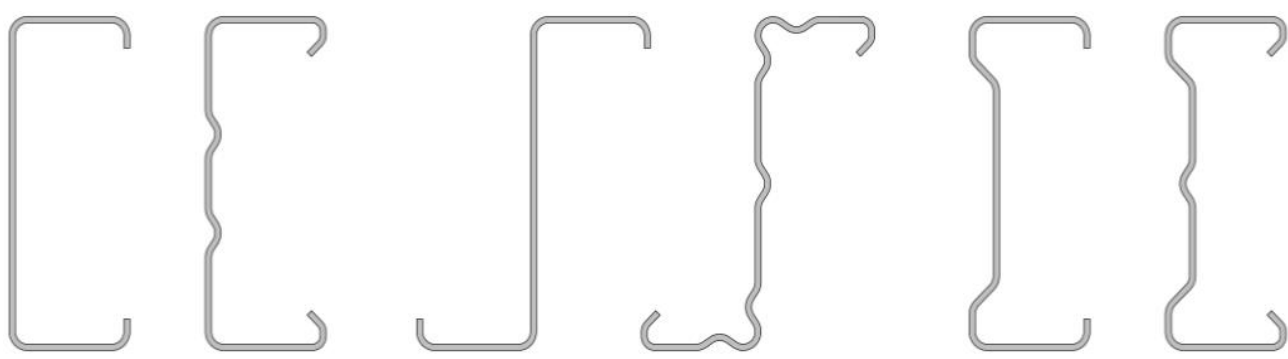

Figure 1: Cold-formed steel $C, Z$ and $\Sigma$ cross-sections with or without stiffeners 


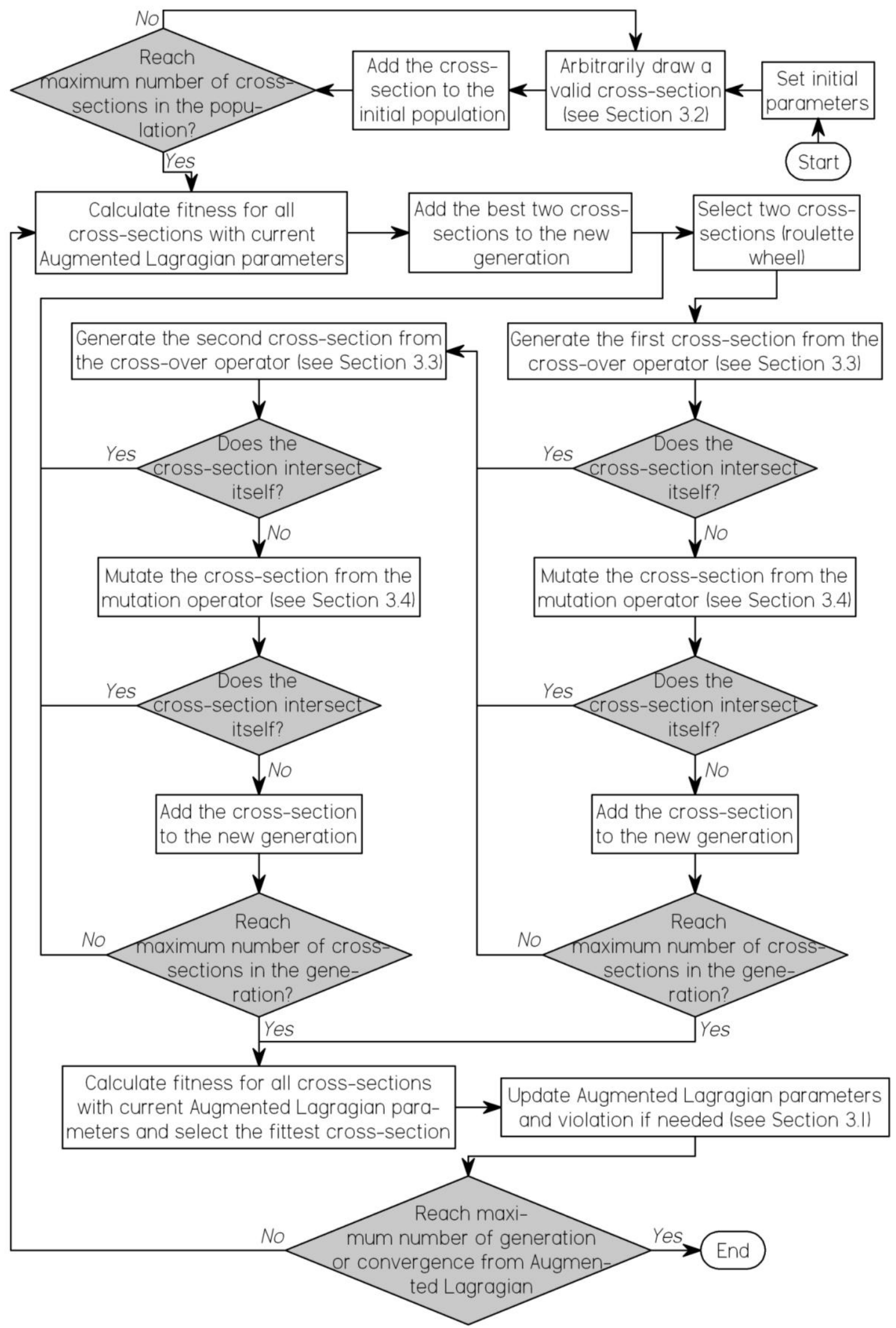

Figure 2: Global flowchart 


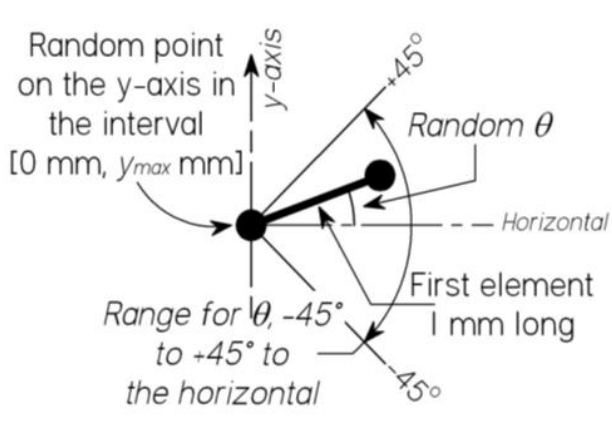

(a)

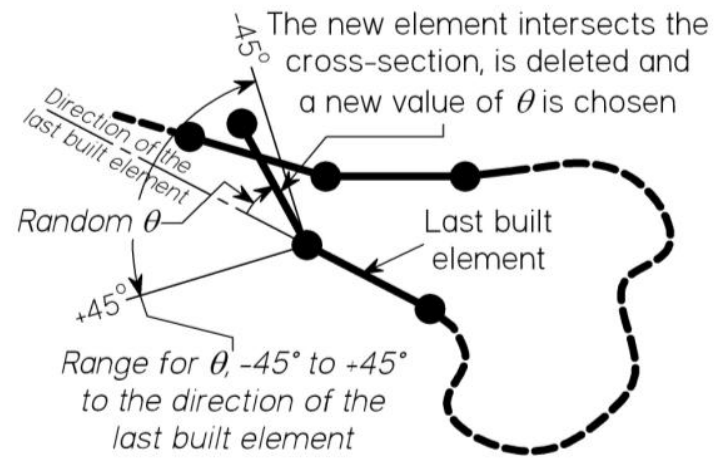

(c)

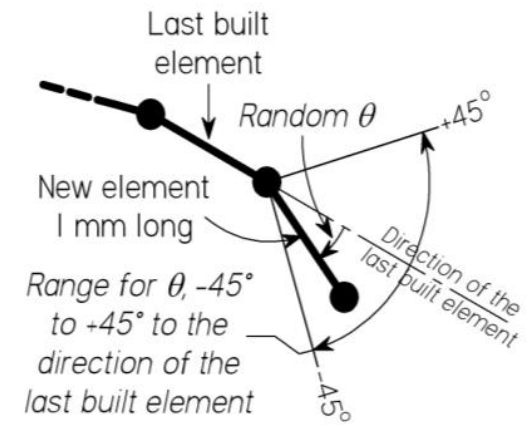

(b)

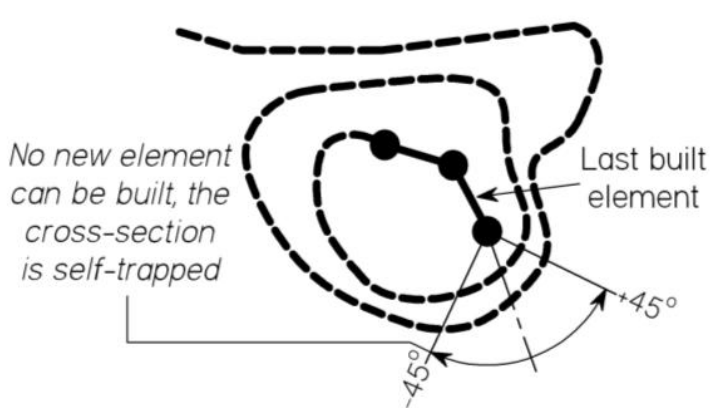

(d)

Figure 3: Creating the initial cross-sections, (a) Step 1, (b) Step 2, (c) Step 3 a) and (d) self-trapped crosssection

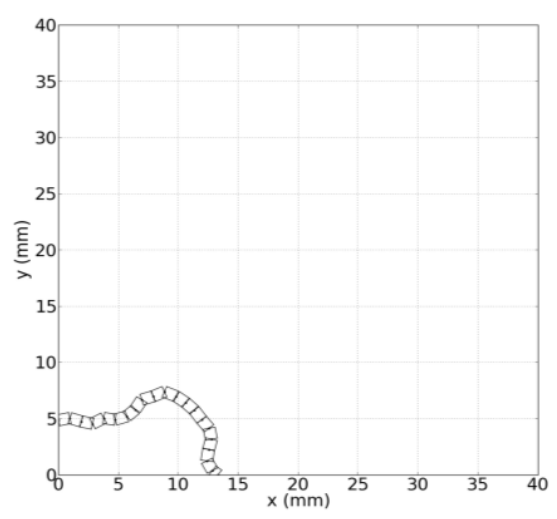

(a)

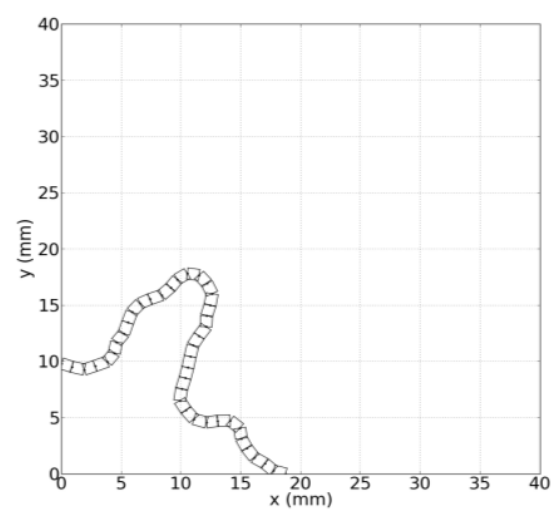

(b)

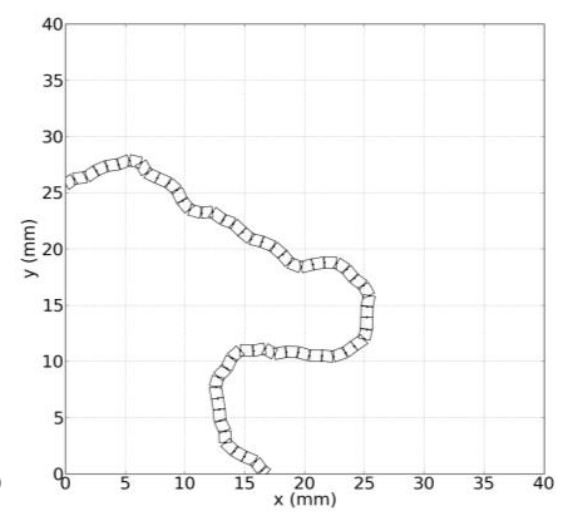

(c)

Figure 4: Initial cross-section on a $40 \mathrm{~mm} \times 40 \mathrm{~mm}$ design space of (a) 20 elements, (b) 40 elements and (c) 60 elements

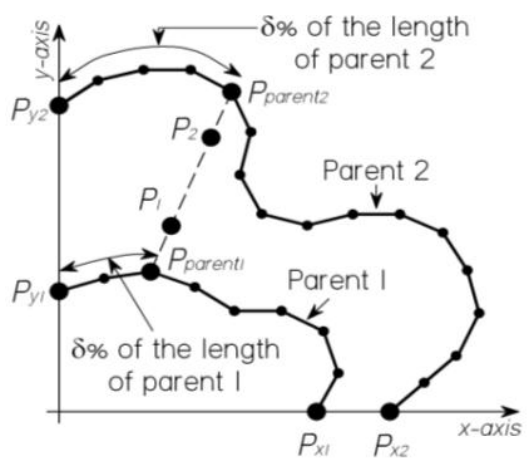

(a)

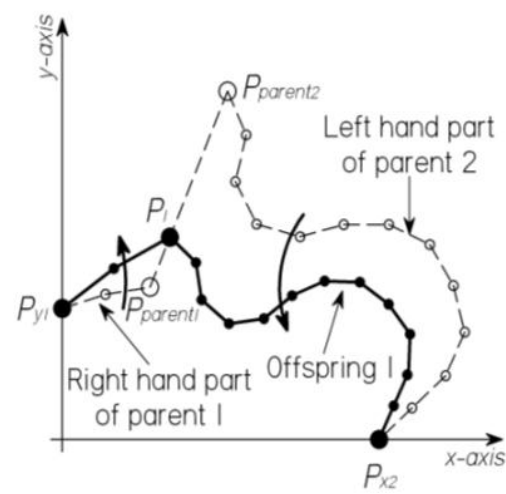

(b)

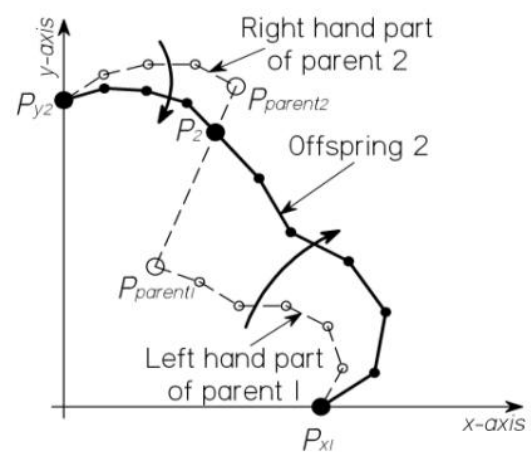

(c)

Figure 5: Cross-over operator (a) defining cross-over points, (b) first offspring and (c) second offspring 


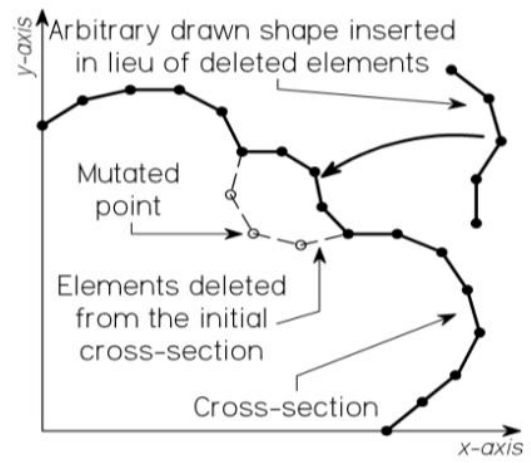

(a)

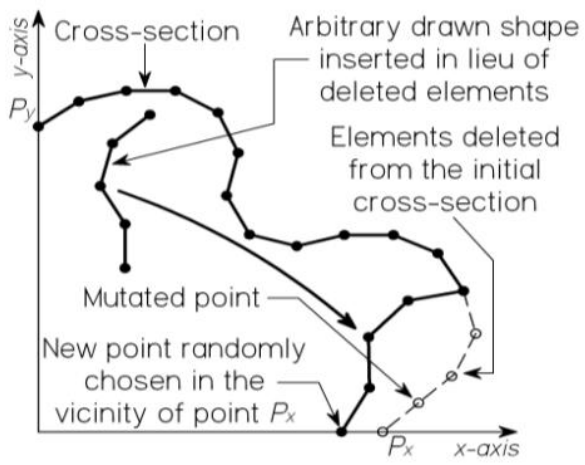

(b)

Figure 6: Mutation operator (a) in the middle part of the cross-section and (b) next to the intersection point $P_{x}$ with the $x$-axis

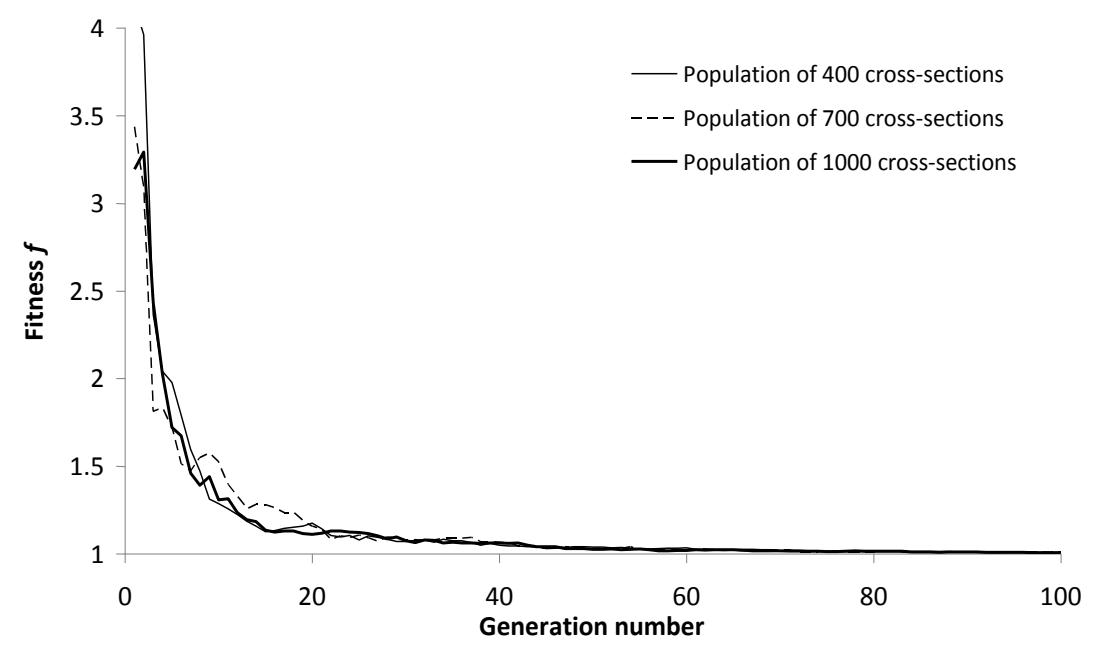

Figure 7: Average fitness ffor various population sizes 


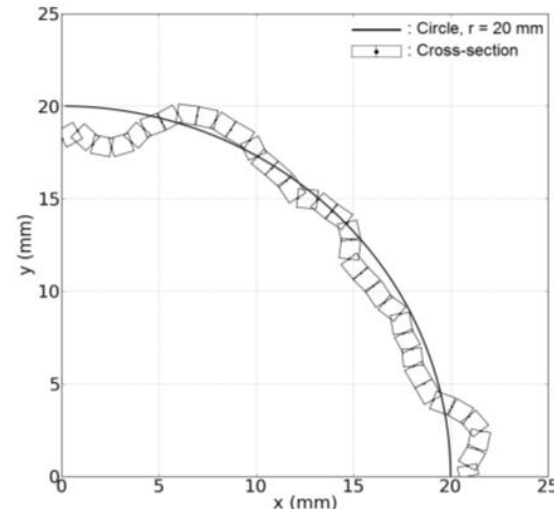

(a) $-1^{\text {st }}$ generation

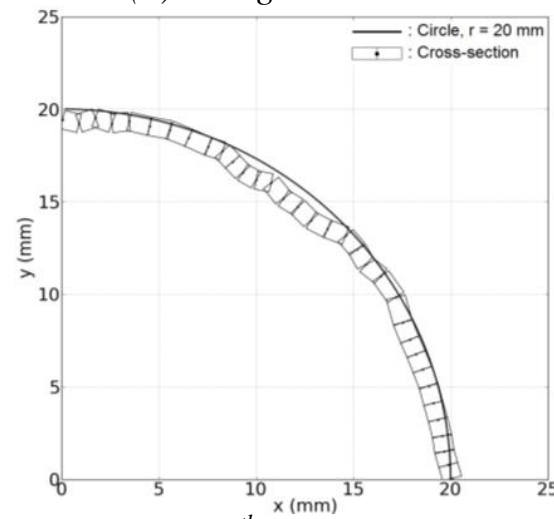

(d) $-15^{\text {th }}$ generation

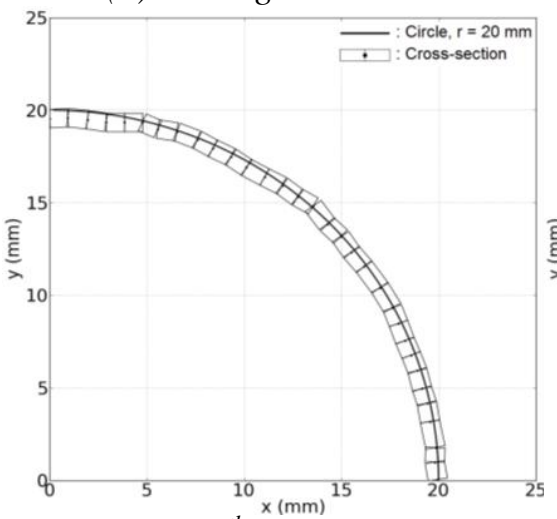

$(g)-35^{\text {th }}$ generation

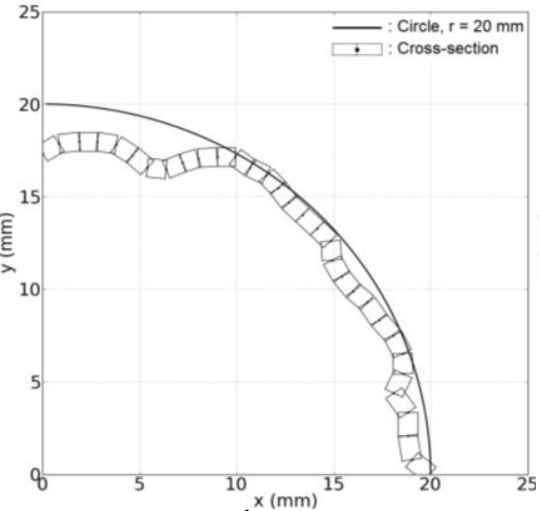

(b) $-5^{\text {th }}{ }^{\mathrm{(mm})}$ generation

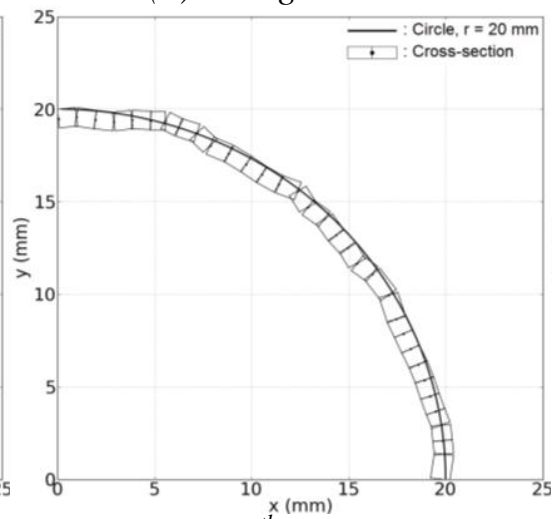

(e) $-20^{\text {th }}$ generation

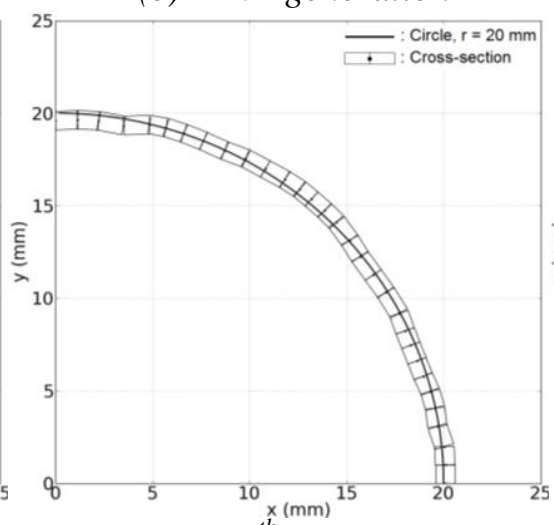

(h) $-60^{\text {th }} \stackrel{x \mathrm{~mm})}{\text { generation }}$

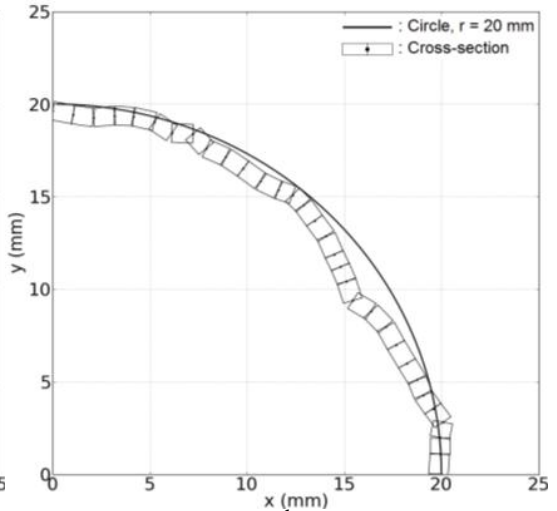

(c) $-10^{\text {th }}$ generation
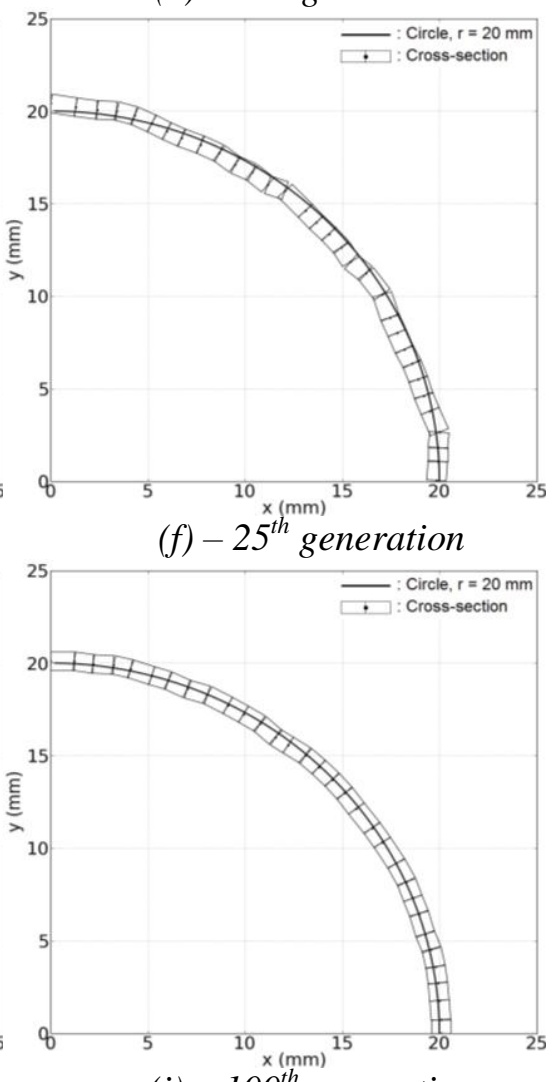

(i) $-100^{\text {th }}$ generation

Figure 8: Fittest cross-sections for a population size of 700 cross-sections from (a) $1^{\text {st }}$ generation (initial population) to $(i) 100^{\text {th }}$ generation 


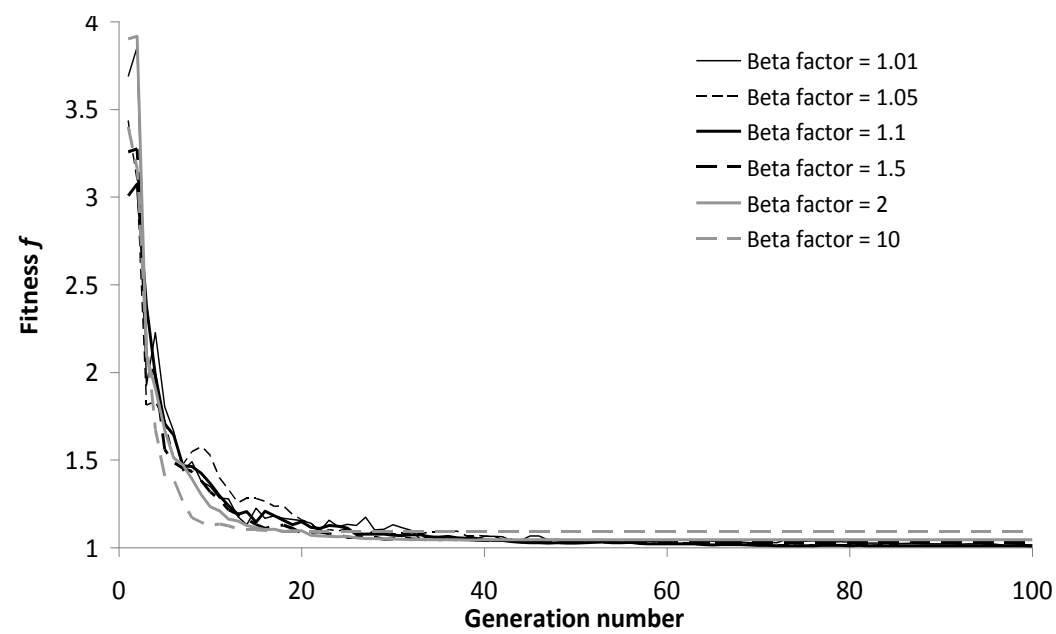

Figure 9: Average fitness $f$ for various $\beta$ factors

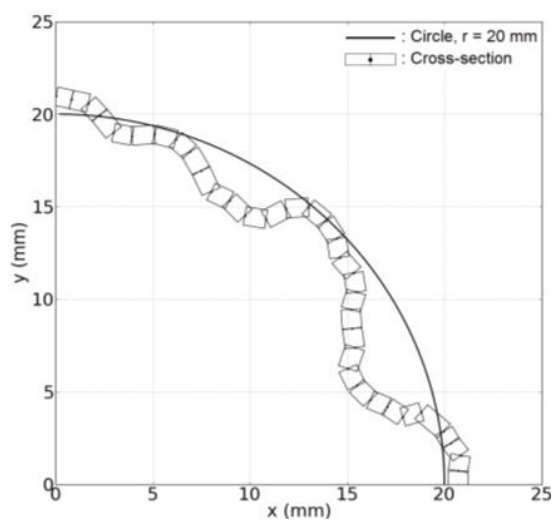

(a)

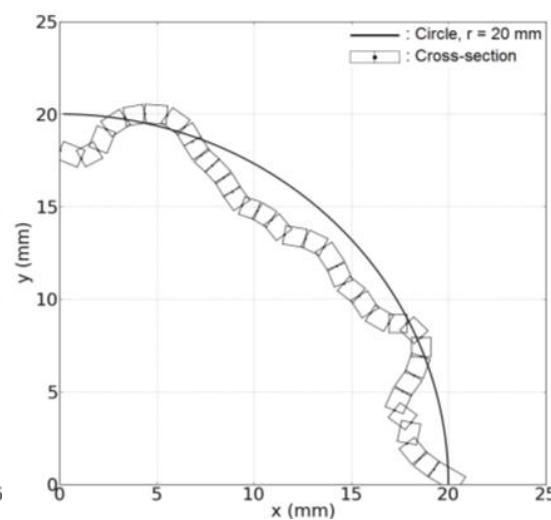

(b)

Figure 10: Typical solutions for penalty increasing constant $\beta=10$ at the $100^{\text {th }}$ generation

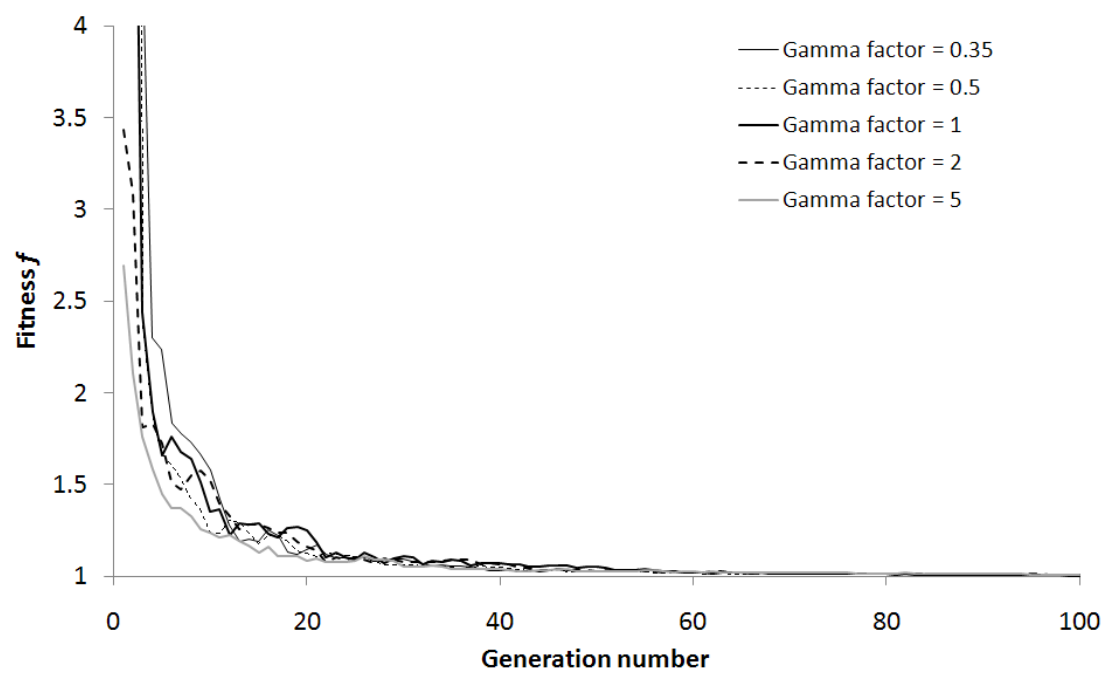

Figure 11: Average fitness f for various penalty function coefficients 


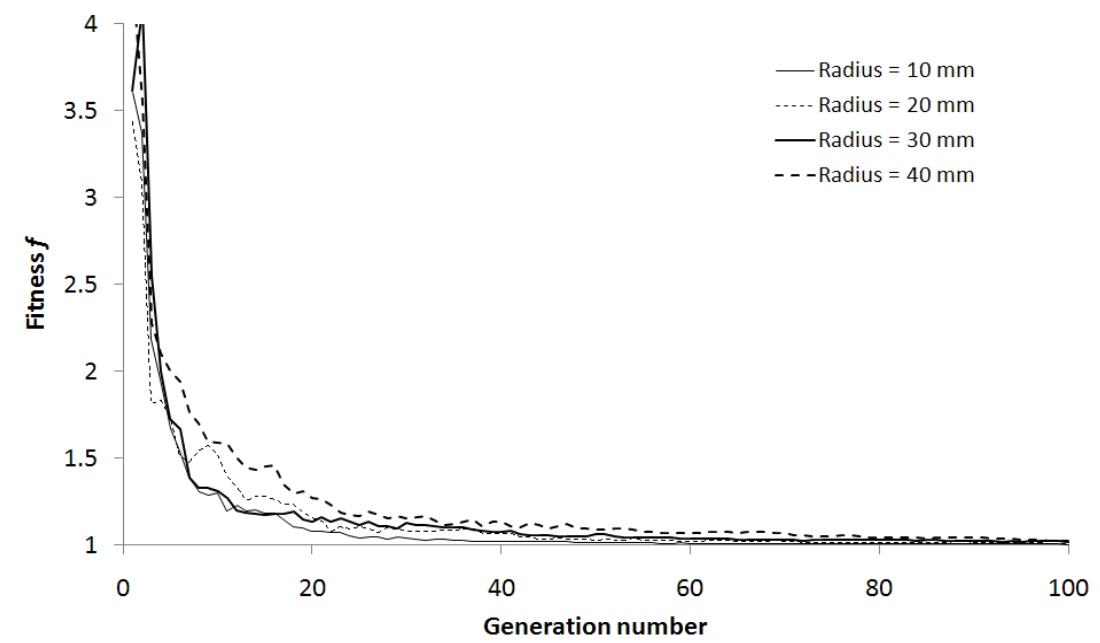

Figure 12: Average fitness ffor various radius circles

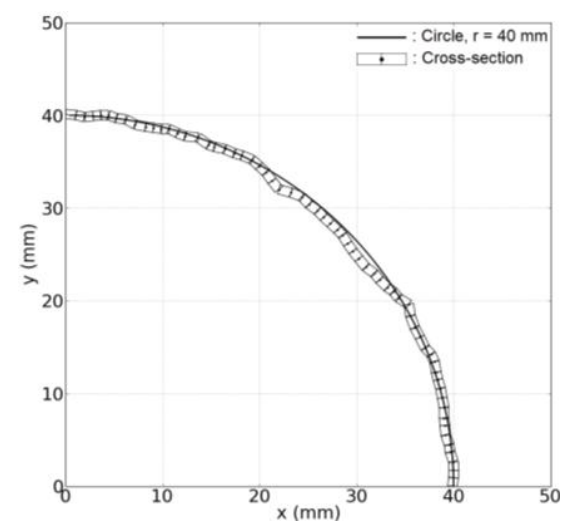

(a)

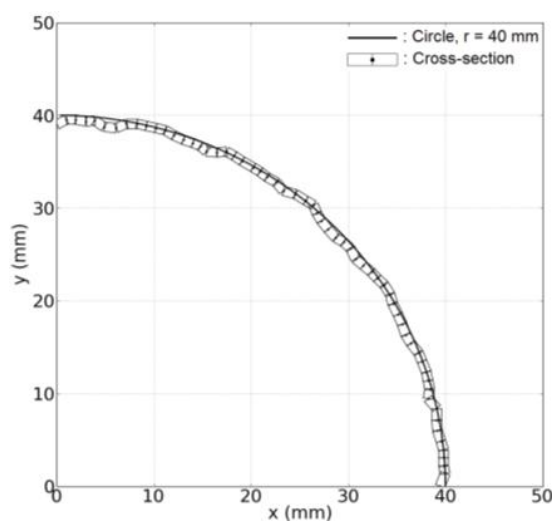

(b)

Figure 13: Typical fittest cross-sections for optimum circle of $40 \mathrm{~mm}$ radius at the $100^{\text {th }}$ generation

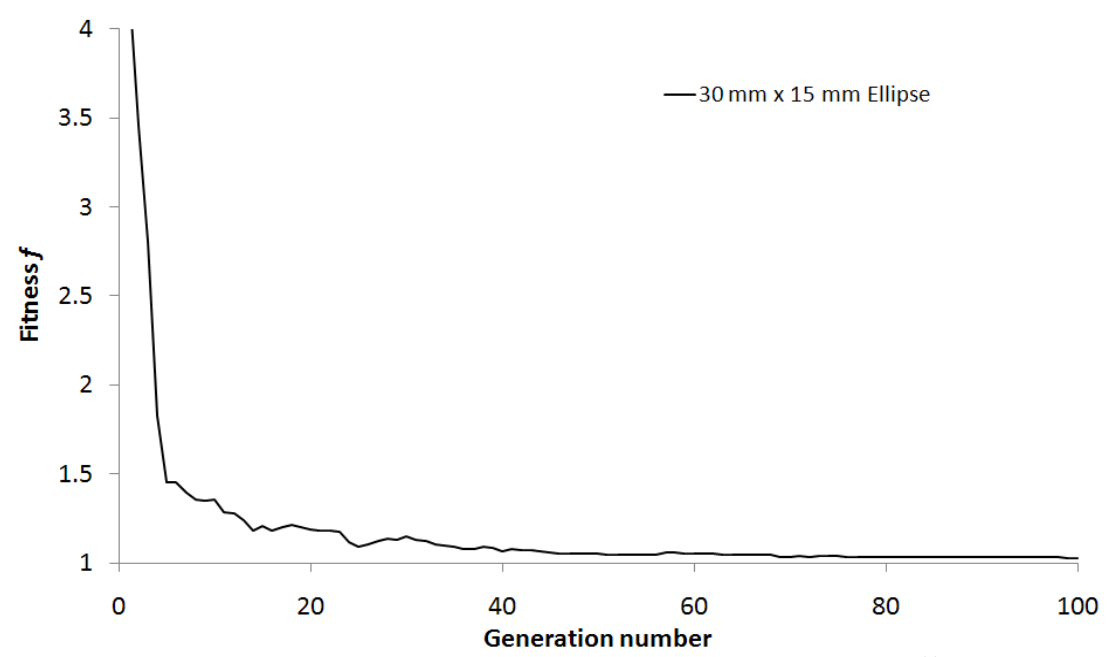

Figure 14: Average fitness for a $30 \mathrm{~mm} \times 15 \mathrm{~mm}$ ellipse 


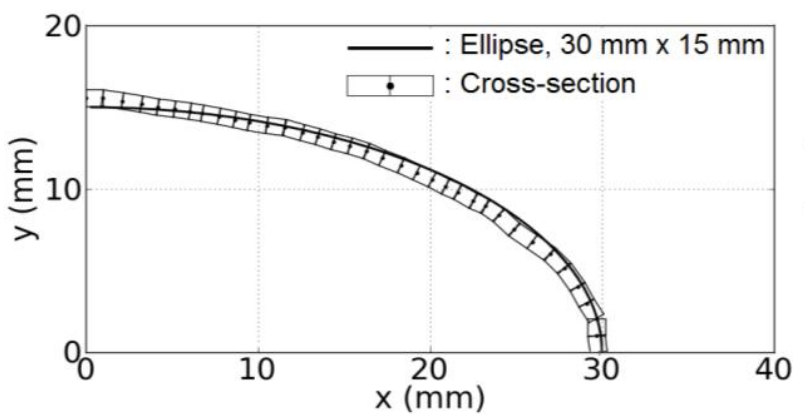

(a)

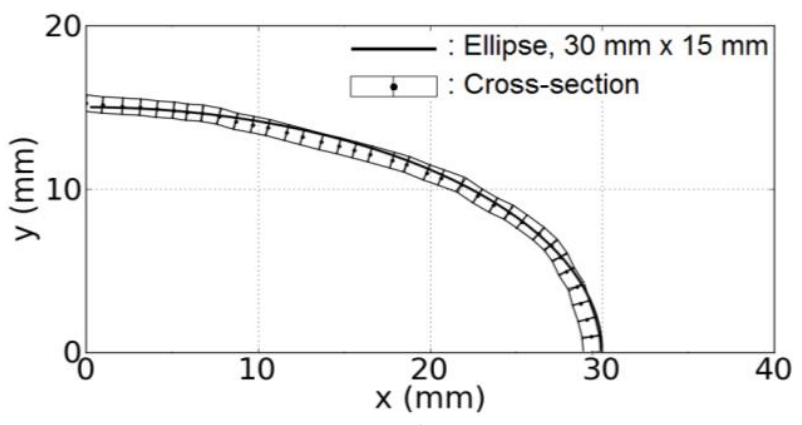

(b)

Figure 15: Typical fittest cross-sections for a $30 \mathrm{~mm} \times 15 \mathrm{~mm}$ ellipse at the $100^{\text {th }}$ generation

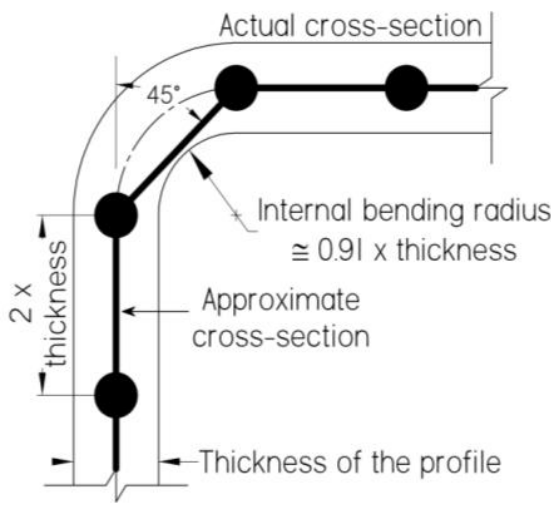

(a)

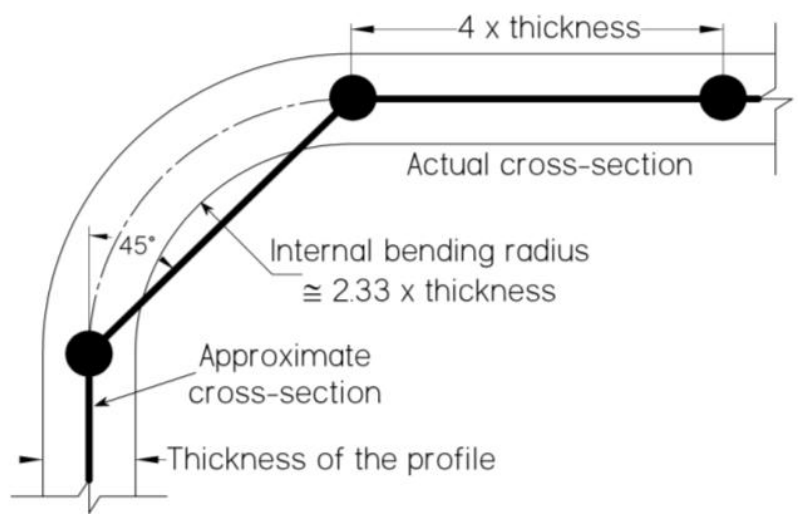

(b)

Figure 16: Minimum Internal bending radius for a $90^{\circ}$ bend in the initial population 